lower ER accumulation of polymeric-F-AT $(\mathrm{P}<0.001)$. In the F-AT cell, PERK mRNA was upregulated at $3 \mathrm{~h}$ compared to $0.5 \mathrm{~h}$ in Z-AT. Although elevated compared to M-AT cells, F-AT cells had lower NF- $\kappa \mathrm{B}$ activity $(\mathrm{P}<0.001)$, TNF-a production $(\mathrm{P}=0.046)$ and IL-6 production $(\mathrm{P}=0.012)$ compared to Z-AT.

In conclusion, F-AT secretion was comparable to M-AT. However, secreted F-AT was defective as an anti-elastase. F-AT was found to polymerise and aggregate in inclusion bodies. ER accumulation of F-AT activated the ER overload response; PERKdependant-NF- $\kappa \mathrm{B}$ mediated inflammatory response, greater than M-AT but to a lesser degree than Z-AT. This data indicate that FZ phenotype may be at risk for liver and lung disease.

\section{S64 CIRCULATING POLYMERS ARE FOUND IN ALPHA-1- ANTITRYPSIN DEFICIENCY AND ARE ASSOCIATED WITH LUNG DISEASE}

${ }^{1} J A$ Dickens, 'L Tan, ${ }^{2} \mathrm{DL}$ DeMeo, ${ }^{3} \mathrm{E}$ Miranda, ${ }^{4} \mathrm{~J}$ Perez, 'ST Rashid, 'J Day, ${ }^{1} \mathrm{~A}$ Ordonez, ${ }^{1} \mathrm{SJ}$ Marciniak, ${ }^{1} \mathrm{I} \mathrm{Haq,}{ }^{5} \mathrm{AF}$ Barker, ${ }^{6} \mathrm{EJ}$ Campbell, ${ }^{7} \mathrm{E}$ Eden, ${ }^{8} \mathrm{NG}$ McElvaney, ${ }^{9} \mathrm{SI}$ Rennard, ${ }^{10} \mathrm{RA}$ Sandhaus, ${ }^{11} \mathrm{JM}$ Stocks, ${ }^{12} \mathrm{JK}$ Stoller, ${ }^{13} \mathrm{C}$ Strange, ${ }^{7} \mathrm{G}$ Turino, ${ }^{14} \mathrm{FN}$ Rouhani, ${ }^{14} \mathrm{M}$ Brantly, 'DA Lomas; 'University of Cambridge, Cambridge, UK; ${ }^{2}$ Brigham and Women's Hospital, Boston, USA; ${ }^{3}$ University of Rome La Sapienza, Rome, Italy; ${ }^{4}$ University of Malaga, Malaga, Spain; ${ }^{5}$ Oregon Health and Science University, Portland, USA; ${ }^{6}$ Intermountain Health Care and Heredilab Inc., Provo and Salt Lake City, USA: ${ }^{7}$ St. Luke's/Roosevelt Hospital, New York City, USA; ${ }^{8}$ Beaumont Hospital, Dublin, Ireland; ${ }^{9}$ University of Nebraska, Omaha, USA; ${ }^{10}$ National Jewish Health, Denver, USA; ${ }^{11}$ University of Texas, Tyler, USA; ${ }^{12}$ Cleveland Clinic, Cleveland, USA; ${ }^{13}$ Medical University of South Carolina, Charleston, USA; ${ }^{14}$ University of Florida, Gainesville, USA

10.1136/thoraxjnl-2013-204457.71

Introduction and Objectives The severe $\mathrm{Z}$ deficiency allele of alpha-1-antitrypsin (Glu ${ }^{342} \mathrm{Lys}$ ) results in the formation of polymers that are retained within hepatocytes, leading to hepatitis, cirrhosis and hepatocellular carcinoma. The concomitant lack of circulating protein predisposes to early onset emphysema. Polymers are also found in the lung, skin and kidney and are known to be proinflammatory, but it is unknown whether those polymers are produced locally or are deposited from a circulating source. We wished to establish whether polymers are present in the plasma of individuals with alpha-1-antitrypsin deficiency, from where they originate and whether they are associated with any clinical phenotype.

Methods We used a novel anti-alpha-1-antitrypsin polymer monoclonal antibody (2C1) in an ELISA assay to evaluate whether polymers are present in a cohort of 513 individuals with ZZ alpha-1-antitrypsin deficiency. Serial samples from an individual with $\mathrm{ZZ}$ alpha-1-antitrypsin deficiency undergoing liver transplantation were used to investigate the source of circulating polymers. We then used a $2^{\text {nd }}$ cohort of 293 individuals with mixed alpha-1-antitrypsin phenotypes to determine whether circulating polymers could be used as a screening test for the presence of a polymerogenic allele. Disease associations were sought using clinical data from the ZZ alpha-1-antitrypsin deficient cohort.

Results In the cohort of 513 individuals with PiZZ alpha-1-antitrypsin deficiency, we found 512 had quantifiable polymers present within serum, the $513^{\text {th }}$ having previously had a liver transplant. Circulating polymer levels were higher in men and individuals with COPD and a there was a correlation with older age and lower lung function. The presence of circulating polymers was $100 \%$ sensitive and $89 \%$ specific in identifying 20 PiZZ alpha-1-antitrypsin homozygotes in a mix of 293 alpha-1antitrypsin genotypes. Serial blood samples from a PiZZ individual undergoing liver transplantation showed that circulating polymers originate from the liver, clearing with a half-life of approximately 30 hours and becoming undetectable 5 days after transplantation.

Conclusions We have shown that circulating polymers are present in PiZZ alpha-1-antitrypsin individuals and originate from the liver. Polymer levels are associated with COPD, suggesting they may play a role in disease pathogenesis.

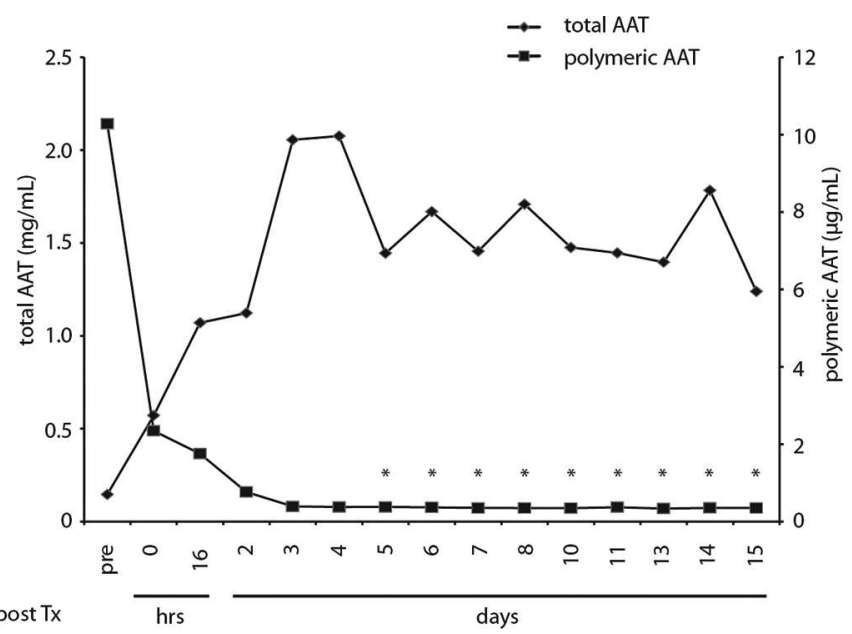

Abstract S64 Figure 1. Z $\alpha 1$ antitrypsin polymers are present in the circulation and are cleared following liver transplantation. Mouse monoclonal antibodies that detect all conformers of $\alpha_{1}$-antitrypsin, or only polymeric $\left(\alpha_{1}\right.$-antitrypsin (2C1), were used to quantify total $\left(\alpha_{1}\right.$-antitrypsin and $\left(\alpha_{1}\right.$-antitrypsin polymers respectively using sandwich ELISA. Time points marked * are below the lower limit of quantification $(0.4 \mathrm{ug} / \mathrm{mL})$.

\section{S65 LARGE AND SMALL AIRWAY EPITHELIAL CELL SENESCENCE PRESENT IN COPD AND BRONCHIECTASIS?}

${ }^{1} \mathrm{~J}$ Birch, ${ }^{2} \mathrm{G}$ Johnson, ${ }^{2} \mathrm{~K}$ Jiwa, ${ }^{1} \mathrm{R}$ Anderson, ${ }^{1} \mathrm{C}$ Ward, ${ }^{1} \mathrm{JJ}$ Taylor, ${ }^{1} \mathrm{PA}$ Corris, ${ }^{1} \mathrm{JF}$ Passos, ${ }^{1}$ AJ Fisher, ${ }^{1} \mathrm{~A}$ De Soyza ; ${ }^{1}$ Newcastle University, Newcastle upon Tyne, UK, ${ }^{2}$ Freeman Hospital, Newcastle upon Tyne, UK

\subsection{6/thoraxjnl-2013-204457.72}

Introduction and Objectives Accelerated lung ageing has been implicated in the pathogenesis of COPD and therefore targeting cellular senescence may have therapeutic benefit. COPD is increasingly felt to have significant sub-phenotypes with large and small airway involvement. The airway epithelium likely endures the majority of potentially senescence-inducing insults. However, data on airway epithelial cell (AEC) senescence in COPD is limited and comparisons between large and small airways are lacking. Furthermore, the role of infection in COPDassociated senescence is unclear. To date, senescence in bronchiectasis has not been investigated as a model for infectioninduced senescence. We sought to determine AEC expression of senescence-associated markers in COPD and bronchiectasis and to compare large and small airways.

Methods Lung explant tissue from our transplant programme from COPD $(n=19)$ and bronchiectasis $(n=14)$ with resection tissue from smokers without lung disease (control) $(\mathrm{n}=11)$ was stained for senescence-associated markers by immunohistochemistry. Staining was quantified semi-quantitatively. Fluorescence in situ hybridisation (FISH) was used to investigate telomere length and possible co-localisation with DNA damage-associated proteins. 